\title{
Parametrical Analysis of Elliptic Flexure Hinges
}

\author{
Yu-juan Chen ${ }^{1, a}$;Jun-sheng jin ${ }^{2, b}$ \\ ${ }^{1}$ Yujuan Chen female, the he bei college of science and technology, No. 1956, south 2nd ring of \\ baoding city, Baoding, China \\ ${ }^{2}$ Jinjun Sheng (1973-9-1), male, south downtown of baoding city , Hebei Province, director of the \\ office of National People's Congress,Baoding, China
}

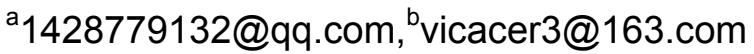

Keywords: compliant mechanism; inherent frequencies; elliptic flexure hinges

\begin{abstract}
The model of parallel-oriented compliant mechanism was established. Analysis of parameters for elliptic flexible hinge plane compliant mechanisms was illustrated. The relationships between the system frequency and the flexible hinges shapes, geometry parameters, material parameters were discussed by numerical examples. The conclusion provides a theoretical basis for elliptic flexible hinges design and application, and also provides references for compliant mechanisms kinetics research.
\end{abstract}

\section{Introduction}

Compliant Mechanism is a new type of mechanical structure which relying on the flexible deformation of itself to output the motion, force or energy. The analysis and synthesis of the compliant mechanism is a new research field relative to the rigid body. Because of there are no motion pair or less, it can be reduced or didn't need to assembly, thus the compliant mechanism have high motion accuracy and greatly improve the motion precision. The compliant mechanism becomes the key component in the fields of bionics, ultra precision positioning mechanism and MEMS et.al [1].

Compliant mechanism can be divided into fully compliant mechanism and partly compliant mechanism. The fully compliant mechanism is all composed of flexible components; and the partly compliant mechanism is composed by both the rigid body and flexible components. Depending on the distribution form of flexible, the fully compliant mechanism can be divided into two types: centralized and distributed, the former is characterized by weaker regions, elastic deformation occurred in the weaker area, it can be used to simulate the movement of hinge; The latter is characterized by no relatively weak areas, and the elastic deformation of flexible component is relatively evenly distributed the entire component [2-4].

Flexible hinge has the advantages of compact structure, small volume, no gap, no friction, no lubrication, smooth continuous movement and high displacement. At present years, it is widely used in the aviation, aerospace, precision measurement and biological engineering. Therefore, the flexible hinge has a broad application prospect in many fields, and there is great significance to study the flexible hinge.

There are two kinds of the common type of flexible hinge: straight beam type flexible hinge and circular arc flexure hinge. Straight beam type flexible hinge have larger rotation range, but the movement precision is not good enough. The circular arc flexure hinge has a good movement precision, but the rotation range is relatively small. In order to consider both the movement precision and the range of movement, some scholars put forward the elliptical flexure hinge [5]. In the engineering design, the numerical integral method and the finite element method (FEM) are the widely used methods.

This paper based on the elliptic flexible hinge, deduced the formula of inherent frequency, and by the use of MATLAB software programming, calculated the relationship between the design parameters and the natural frequency under the condition of the deflection angle, and get the 
influence law of various design parameters on the system frequency characteristics though the comparison of the calculated results. It can be provide theoretical basis for design and use the elliptic flexible hinge in application.

\section{Flexible Hinge Parallel Orientation Mechanism Model}

Flexible hinge parallel orientation mechanism is shown in Fig.1. In the process of deformation, connecting rod and the connecting support rod assumed rigid parts, the deformation can be neglect, flexible hinge bent and its elastic deformation related to the stiffness $\mathrm{K}$. Dynamics analysis of this flexible hinge parallel orientation mechanism as follows:

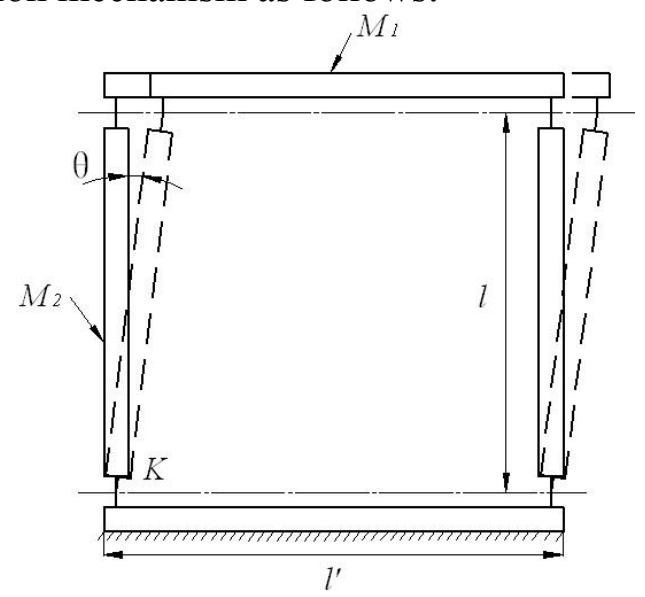

Fig. 1. Simplified model of flexible hinge parallel orientation mechanism Translational energy of connecting rod:

$$
T_{1}=\frac{1}{2} M_{1}(l \dot{\theta})^{2}
$$

Total kinetic energy of the two connecting support rod:

$$
T_{2}=2\left[\frac{1}{2} J \dot{\theta}^{2}+\frac{1}{2} M_{2}\left(\frac{1}{2} l \dot{\theta}\right)^{2}\right]
$$

The elastic deformation energy of four flexure hinge:

$$
U=\frac{1}{2}\left(K_{1}+K_{2}+K_{3}+K_{4}\right) \theta^{2}=2 K \theta^{2}
$$

Here, in order to convenient the study, assume that:

$$
K_{1}=K_{2}=K_{3}=K_{4}=K
$$

System Lagrange equation:

$$
L=\frac{1}{2} M_{1}(l \dot{\theta})^{2}+J \dot{\theta}^{2}+\frac{1}{4} M_{2}(l \dot{\theta})^{2}-2 K \theta^{2}
$$

System dynamics equation:

$$
\left(M_{1} l^{2}+2 J+\frac{1}{2} M_{2} l^{2}\right) \ddot{\theta}+4 K \theta=0
$$

where $E$ is the elastic modulus of the material, $I$ is the section moment of inertia, $J$ is the moment of inertia of the connecting support rod around the center of mass, $l$ is the total length of the rod, $M_{l}$ is the quality of the connecting rod, $M_{2}$ is the quality of the connecting support rod, $\theta$ is the deflection angle, $K_{i}(i=1,2,3,4)$ is the equivalent torsion spring coefficient. 


\section{Frequency Response Analysis}

System inherent frequency is one of the important evaluation indicators, it has great significance on the system dynamic performance optimization, control and optimization design et.al. The system inherent frequency $f$ is shown as Eq.7:

$$
f=\frac{\omega}{2 \pi}=\frac{1}{2 \pi} \sqrt{\frac{4 K}{M_{1} l^{2}+2 J+\frac{1}{2} M_{2} l^{2}}}
$$

First, the calculation formula of $K$ is associated with the shape of the elliptic flexure hinge:

$$
\begin{gathered}
K=\frac{E w b^{3}}{12 a f_{3}} \\
f=\int_{\pi / 2}^{-\pi / 2} \frac{\cos \theta}{\left(\frac{t}{b}+2-2 \cos \theta\right)^{3}} d \theta
\end{gathered}
$$

The parameters are shown in Fig.2.

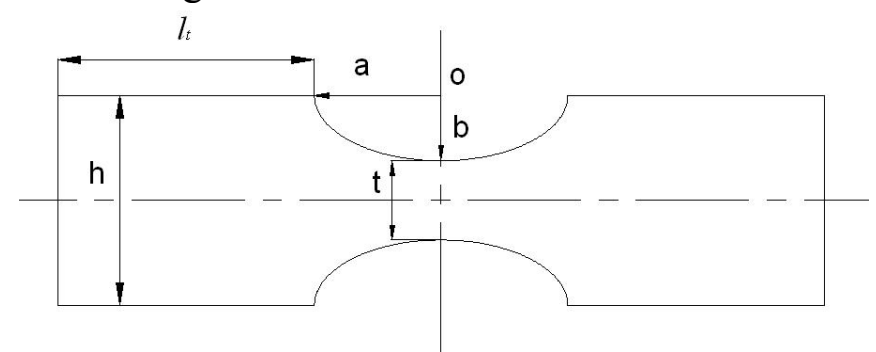

Fig. 2. Elliptic flexure hinge

Second, the calculation formulas of M1 and M2 are all contain the geometric parameters of the elliptic flexure hinge, and they are shown as Eq.10 and Eq.11.

$$
\begin{gathered}
M_{1}=\left(h l^{\prime}+2 h l_{t}-\pi a b\right) \rho w \\
M_{2}=(h l-\pi a b) \rho w
\end{gathered}
$$

Third, the calculation of the moment of inertia for the connecting support rod around the center of mass $\mathrm{J}$ is shown as Eq.12. Here, on the assumption that the deformation of the elliptic flexible hinge is turning around its geometric center, ignore the displacement caused by the flexible hinge deformation. So the formula of moment of inertia is:

$$
J=\frac{1}{3} M_{2} l^{2}
$$

We substitute Equations (8) (12) into Equation (7) to obtain a final expression for the system inherent frequency of the elliptic flexure hinge compliant mechanism, the $f$ is relation to the parameters both on geometric and material.

$$
f=\frac{1}{2 \pi} \frac{1}{l} \sqrt{\frac{E}{\rho}} \sqrt{\frac{R^{2}}{f_{3}\left(3 h l^{\prime}+\frac{7}{2} h l+6 h l_{t}-\frac{13}{2} \pi a b\right)}}
$$

By the Eq.13 we can get the following conclusion: 
1. When the section parameters and material parameters of the mechanism is determined, the system inherent frequency of elliptic flexure hinge compliant mechanism are closely related to the parameters of $t, a$ and $b$.

2. The system inherent frequency is not related with the thickness $w$.

\section{Numerical Calculation And Analysis}

In order to simplified the numerical calculation, when change one of the parameters of compliant mechanisms, the other parameters are keep the same. And this paper only calculating first-order inherent frequency values.

Fig. 6 is the finite element model established by ANSYS. The geometry parameters are set as: $L_{1}=50 \mathrm{~mm}, L_{2}=30 \mathrm{~mm}, w=10 \mathrm{~mm}, h=5 \mathrm{~mm}$, the material is polypropylene, and its density is $0.90 \mathrm{~g} / \mathrm{cm}^{3}$, Poisson's ratio is 0.49 , modulus of elasticity is $E=1.42 \mathrm{GPa}$. The element choose Structural solid Brick 20node186 unit to free agency. The completely displacement constraints are added to the bottom node.

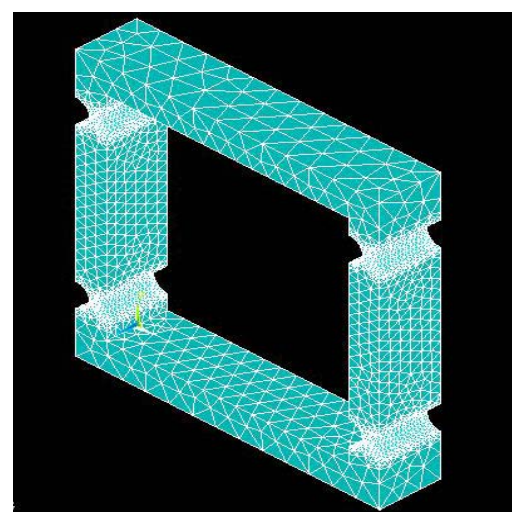

Fig. 3. Finite element model

The Relationship Between Inherent Frequency And Minimum Thickness .Fig.4 shows the relationship between the system inherent frequency and minimum thickness, it can be concluded that, with the increase of the design parameters $t$, the first order inherent frequency of compliant mechanisms are nonlinear increase. This can be thought of as the design parameter $t$ increase its stiffness increases, which is good to improve the motion precision and reduce the vibration of compliant mechanisms. But the flexibility of the system is reduced which bring a great influence to the operational flexibility.

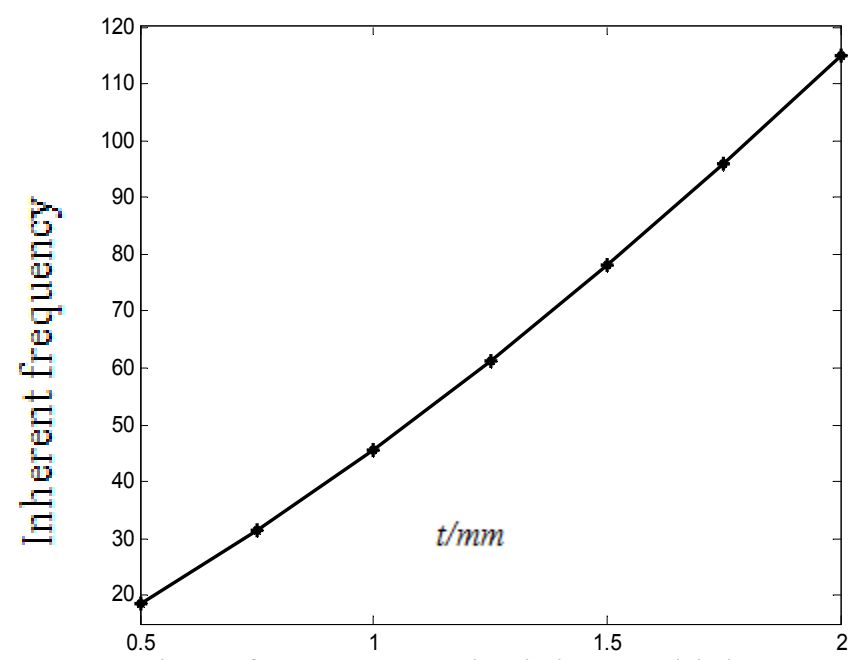

Fig. 4 frequency and minimum thickness $t$

It also can be seen from the Fig. 4 that, the relationship between the inherent frequency of elliptical flexible hinge parallel orientation mechanism and minimum thickness is increased progressively, and the growth speed slowly greater. 
The Relationship Between Inherent Frequency And The Incision Parameters. Fig.5 represents the relationship between the system inherent frequency and the incision parameters such as the long half axis $a$ and the short half axis $b$. It can be seen that, the inherent frequency of elliptical flexible hinge mechanism is decreasing with the increase the long half axis $a$, the decrease speed are gradually lower; the inherent frequency of elliptical flexible hinge mechanism is increasing with the increase of the short half axis $b$, but the amplification is small.

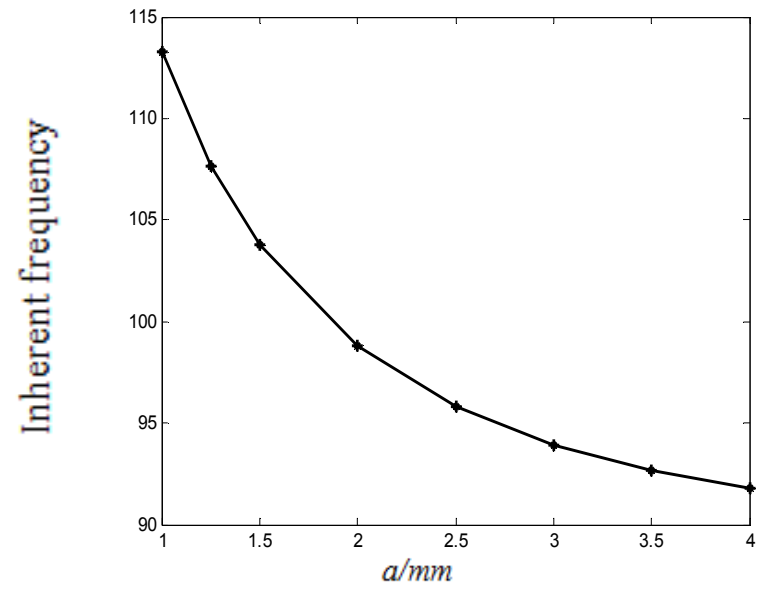

a. long half axis $a$

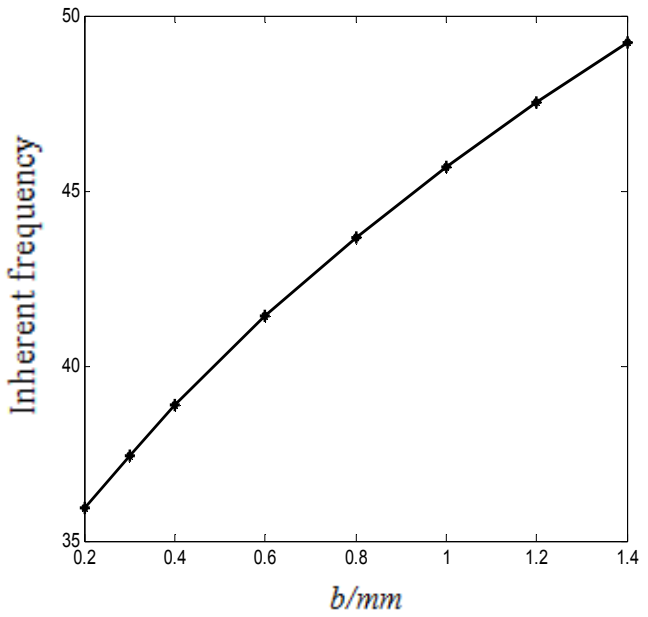

b. short half axis $b$

Fig. 5 The relationship between the system inherent frequency and the incision parameters

The Relationship Between Inherent Frequency And The Thickness Of Mechanism. Under the condition of other parameters constant to change the thickness of mechanism $w$, the Fig. 6 shows that with the increase of the thickness, the system inherent frequency remains unchanged, so the thickness of mechanism $w$ has no effect on the first order of inherent frequency. This is anatomize to the theoretical analysis.

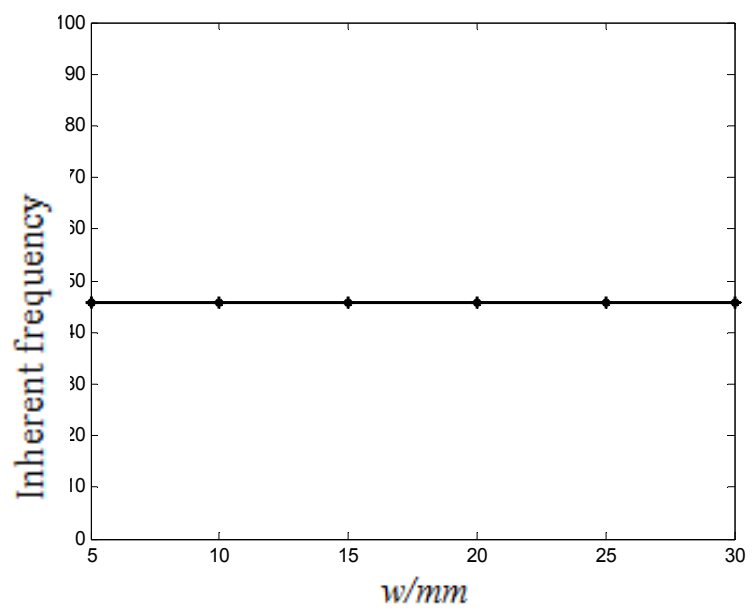

Fig. 6 frequency and the thickness of $w$

\section{Conclusions}

Through the calculation and analysis above, we can get that, the system inherent frequency of elliptical flexible hinge parallel orientation mechanism was biggest influenced by the minimum thickness $t$, second of long half axis $a$, third of short half axis $b$, and no effect from the thickness of the mechanism $w$. In practical application, the rule in this paper has a guiding significance in the design or select of elliptical flexure hinge. 


\section{References}

[1] Howell L. L. Compliant Mechanism. New York: McGrawHill, 2001

[2] Kota S, Jinyong Joo, Li Zhe, Steven M. Rodgers, Jeff Niegowski. Design of Compliant Mechanisms: Applications to MEMS. Analog Integrated Circuits and Signal Processing, 2001, 29: $7 \sim 15$

[3] Midha A, Norton T. W, Howell L. L. On the Nomenclature, Classification, and Abstractions of Compliant Mechanisms.ASME Journal of Mechanical Design, 1994, Vol. 116(1): 270 279

[4] Hetrick J. A. An Energy Efficiency Approach for Unified Topological and Dimensional Synthesis of Compliant Mechanisms.Michigan: University of Michigan, 1999

[5] Smith S. T, Badam I. V. G, Dale J. S. Elliptical flexure hinges[J]. Review of Scientific Instruments, 1997, 68(3):1474 1483 